\title{
Magnetically induced vacuum decay
}

\author{
She-Sheng Xue* \\ ICRA, INFN and Physics Department, \\ University of Rome "La Sapienza", 00185 Rome, Italy
}

\begin{abstract}
We study the fermionic vacuum energy of vacua with and without being applied an external magnetic field. The energetic difference of two vacua leads to the vacuum decaying and the vacuum-energy releasing. In the context of quantum field theories, we discuss why and how the vacuum energy can be released by spontaneous photon emissions and/or paramagnetically screening the external magnetic field. In addition, we quantitatively compute the vacuum energy released, the paramagnetic screening effect and the rate and spectrum of spontaneous photon emissions. The possibilities of experimentally detecting such an effect of vacuum-energy releasing and this effect accounting for the anormalous X-ray pulsar are discussed.

PACS numbers: $12.20 \mathrm{ds}, 12.20 \mathrm{fv}$
\end{abstract}

\section{INTRODUCTION}

The vacuum has a very rich physical content in the context of relativistic quantum field theories. It consists of extremely large number of virtual particles and anti-particles. The quantum-field fluctuations of the vacuum are creations and annihilations of these virtual particles and anti-particles in all possible energy-range. As a consequence of the quantum fluctuations of bosonic and fermionic fields in the vacuum, the vacuum energy does not vanish. In quantum field theories for free and massless particles, the positive vacuum-energy (the zero-point energy) of virtual photons is given by,

$$
\mathcal{E}_{o}=2\left(\frac{V}{(2 \pi)^{3}}\right) \int d^{3} p \epsilon(|p|), \quad \epsilon(|p|)=\sqrt{p_{x}^{2}+p_{y}^{2}+p_{z}^{2}}
$$

in a volume $V$ of the three-dimensional space, where the factor "2" is for polarization states. Analogously, with the negative and non-degenerate energy-spectrum of free virtual fermions,

*Electronic address: xue@icra.it 
the fermionic vacuum energy is given by

$$
\begin{aligned}
\mathcal{E}_{o} & =4\left(\frac{V}{(2 \pi)^{3}}\right) \int d^{3} p\left|\epsilon_{F}(p)\right|, \\
\epsilon_{F}(|p|) & =-\sqrt{p_{x}^{2}+p_{y}^{2}+p_{z}^{2}+m^{2}},
\end{aligned}
$$

where $m$ is the mass of fermions and the factor " 4 " is for spin states. In Eqs.(12), the summation is over all possible momentum-states of quantum-field fluctuations. Up to the fundamental Planck scale $\Lambda_{p}$, the vacuum-energy Eqs.(12) are constants, $\left|\mathcal{E}_{o}\right| \sim V \Lambda_{p}^{4}$.

In the description of renormalizable and perturbative quantum field theories, the vacuum state as ground state is that all negative energy states are fully filled by the pairs of virtual fermions and anti-fermions. The virtual fermion in the negative energy state travels backwards in time indicating a virtual anti-fermion in the positive energy state travels forwards in time. The pairs of virtual fermions and anti-fermions are created by virtual photons and annihilated into virtual photons in the time scale $\sim \hbar / m c^{2}$ and at the distance scale $\sim \hbar / m c$. While, real particles and antiparticles are excitation quanta upon the vacuum state. The vacuum energy Eqs.(12) are dropped and set to be zero by the normal ordering of creation and annihilation operators, owing to the absolute value of the physical energy only determined up to a constant. The quantum-field fluctuations of the vacuum impacting on real particles and antiparticles are treated by the renormalization of quantum field theories. The descriptions of renormalizable and perturbative quantum field theories have been extremely successful, as examples, the Lamb-shift effect [1] and electric charge renormalization. These effects indeed exhibit the highly non-trivial structure of the quantum electromagnetic dynamics (QED) and its vacuum (ground) state.

However, as shown by the Casimir effect 2] that was experimentally evidenced [3], the positive vacuum-energy (10) of virtual photons is not just a trivial constant, when the quantum fluctuations of virtual photons of the vacuum state are confined within a finite volume by boundary conditions. This effect shows that the vacuum state is modified by boundary conditions. From the energetical point of view, the Casimir effect can be physically understood as the following: (i) the continuous energy-spectrum (11) of electromagnetic fields is modified by boundary conditions to be discrete one; (ii) the vacuum energy of "final" vacuum state, computed by the discrete energy-spectrum in a given finite volume $V$, is smaller than the vacuum energy of "initial" vacuum state, computed by the continuous energy-spectrum (11) in the same volume; (iii) as a result, the vacuum gains energy and becomes energetically 
unstable and has to decay from the "initial" vacuum state to the "final" vacuum state by quantum-field fluctuations. The difference of vacuum energies between two vacuum states must be released and this leads to an attractive and macroscopic force observed in the Casimir effect.

In this article, instead of modifying the energy-spectrum (11) of virtual photons by boundary conditions, we attempt to study the variation of the vacuum-energy (2) by modifying the negative energy-spectrum (3) of virtual fermions by an external magnetic field. We try to find any possible observable effects of the vacuum decay due to such a modification, and discuss the possibilities that these effects could be experimentally tested.

\section{VACUUM INSTABILITY}

Within a space volume $V=L_{x} \cdot L_{y} \cdot L_{z}$, we introduce an external constant magnetic field $B$ along the z-axis. As well known as the Landau levels 4$]$, the negative energy-spectrum of virtual charged fermions is given by

$$
\epsilon\left(p_{z}, n, \alpha\right)=-\sqrt{p_{z}^{2}+m^{2}+|e| B(2 n+1)-e B \alpha}, \quad n=0,1,2,3, \cdots,
$$

where $e$ and $\alpha= \pm 1$ are fermion's bare charge and helicity. This negative energy-spectrum is degenerate in the phase space of $\left(p_{x}, p_{y}\right)$ and the degeneracy is $|e| B S /(2 \pi)$, where the area $S=L_{x} \cdot L_{y}$.

We define the vacuum state with $B=0$ as "initial" vacuum state and the vacuum state with $B \neq 0$ as "final" vacuum state. The negative energy-spectrum (3) of "initial" vacuum state is modified to the negative energy-spectrum (4) of "final" vacuum state, due to the external magnetic field $B$. If the vacuum energy of the "final" vacuum state made by virtual fermions fully filling the negative energy-spectrum (4) is smaller than that of the "initial" vacuum state made by virtual fermions fully filling the negative energy-spectrum (3), the vacuum state gains energy and must decay from the "initial" vacuum state to the "final" vacuum state by quantum-field fluctuations. The difference of vacuum energies between two vacuum states must be released, leading to possibly observable effects. In order to verify this, we are bound to compute the energetic difference between two vacuum states respectively corresponding to $B=0$ and $B \neq 0$.

The vacuum energy of the "initial" vacuum state $(B=0)$ is given by eq.(2). Whereas, 
the vacuum energy of the "final" vacuum state $(B \neq 0)$ is given by,

$$
\mathcal{E}_{n}=-\left(\frac{|e| B S}{2 \pi}\right)\left(\frac{L_{z}}{2 \pi}\right) \int d p_{z} \sum_{n, \alpha}\left|\epsilon\left(p_{z}, n, \alpha\right)\right| .
$$

Both vacuum energies (2) and (15) are divergent up to the Planck scale $-V \cdot \Lambda_{n .}^{4}$ By using the approaches of the dimensional regularization [5] and $\xi$-function regularization [6], we compute the vacuum energies Eq.(2) for $B=0$ and Eq.(15) for $B \neq 0$. In Eq.(2), analytically continuing the dimension of the momentum integration from 3 to $3+\epsilon$, where $\epsilon$ is a small complex parameter, we have

$$
\mathcal{E}_{o}=\left(\frac{V \pi}{(2 \pi)^{3}}\right) m^{4} \Gamma\left(-\frac{\epsilon}{2}\right),
$$

where $\Gamma(x)$ is the Gamma-function. Analogously, in Eq.(15), analytically continuing the dimension of the momentum integration from 1 to $1+\epsilon$, we have,

$$
\mathcal{E}_{n}=-\left(\frac{|e| B V}{4 \pi^{2}}\right) \Gamma\left(-\frac{\epsilon}{2}\right) \sum_{n, \alpha}\left[m^{2}+|e| B(2 n+1)-e B \alpha\right]^{z}
$$

where $z=1+\epsilon / 2$. Summing over helicity states $\alpha= \pm 1$ in Eq.(17), we obtain

$$
\begin{aligned}
\mathcal{E}_{n} & =-\left(\frac{|e| B V}{4 \pi^{2}}\right) \Gamma\left(-\frac{\epsilon}{2}\right) \sum_{n}\left[\left(m^{2}+2|e| B n\right)^{z}+\left(m^{2}+2|e| B(n+1)\right)^{z}\right] \\
& =-\left(\frac{2|e|^{2} B^{2} V}{4 \pi^{2}}\right) \Gamma\left(-\frac{\epsilon}{2}\right)[\xi(-z, q)+\xi(-z, q+1)], \quad q=\frac{m^{2}}{2|e| B}
\end{aligned}
$$

where $\xi(z, q)$-function is given by Eqs.(9.521), (9.531) and (9.627) in [7],

$$
\xi(z, q)=\sum_{n=0} \frac{1}{(n+q)^{z}}, \quad \xi(-1, q)=-\frac{B_{2}(q)}{2}, \quad B_{2}(q)=q^{2}-q+\frac{1}{6} .
$$

The analytic continuation " $z$ " has simply discarded the appropriate divergent terms and the continuation back to " $z=1$ " $(\epsilon \rightarrow 0)$ yields,

$$
\mathcal{E}_{n}=V \Gamma\left(-\frac{\epsilon}{2}\right)\left[\frac{|e|^{2} B^{2}}{3\left(4 \pi^{2}\right)}+\frac{\pi\left(m^{2}\right)^{2}}{(2 \pi)^{3}}\right]
$$

These results Eq.(6) for $B=0$ and Eq.(10) for $B \neq 0$ are in agreement with Eqs.(3.8) and (4.2) in the ref. [8]. This provides us a consistent check of our computations.

As a result, the energetic difference of the "initial" vacuum state $(B=0)$ and "final" vacuum state $(B \neq 0)$ is,

$$
\Delta \mathcal{E}=\mathcal{E}_{n}-\mathcal{E}_{o}=\Gamma\left(-\frac{\epsilon}{2}\right)\left[\frac{|e|^{2} B^{2} V}{3\left(4 \pi^{2}\right)}\right]
$$


We find that in Eq. (10) the term depending on the fermion mass is completely canceled by Eq.(6), as it should be. For $\epsilon \rightarrow 0$, the Gamma-function $\Gamma(-\epsilon / 2)=-(2 / \epsilon+$ const. $)$, where the constant is an uninteresting combination of $\pi, \gamma$ (Euler constant), etc. On the basis of the charge renormalization of the QED, we renormalize the charge $\left|e_{r}\right|=\sqrt{Z_{3}}|e|$ by the renormalization constant $Z_{3}=(2 / \epsilon+$ const. $)$ and we obtain,

$$
\Delta \mathcal{E}=-\sum_{f}\left(Q_{f}^{2}\right) \frac{e_{r}^{2} B^{2} V}{3\left(4 \pi^{2}\right)}=-8 \frac{\alpha}{3 \pi} B^{2} V, \quad \alpha=\frac{e_{r}^{2}}{4 \pi}=\frac{1}{137},
$$

where $\sum_{f}\left(Q_{f}^{2}\right)=8$ for all charged fermions in the standard model of particle physics. Eq. (12) is about one percent of the total energy deposited by the external magnetic field $B$.

The energetic difference Eq. (12) between the vacuum states $(B=0)$ and $(B \neq 0)$ is negative, indicating that the vacuum energy Eq.(6) $(B \neq 0)$ is smaller than the vacuum energy Eq.(10) $(B=0)$, i.e., the vacuum state gains energy when the external magnetic field is applied upon it. The reasons are following. (i) In a finite volume $V$ and the finite momentum-cutoff at the Planck scale $\Lambda_{p}$, the total number of fermion states in the vacua of negative energy-spectra (3) and (44) are finite and all these fermion states of negative energy levels from $-\Lambda_{p}$ to $-m c^{2}$ are fully filled. (ii) The negative energy-spectrum (3) is not degenerate, while the negative energy-spectrum (44) is degenerate, and the total numbers of fermion states of both cases are the same. (iii) On the basis of quantum-field fluctuations towards the lowest energy-state and the Pauli principle, when the external magnetic field $B$ is applied upon the vacuum, the vacuum reorganizes itself by fully filling all fermion states according to the degenerate negative energy-spectrum (4), instead of the non-degenerate one (3). As a consequence, the vacuum makes its total energy lower. As an analogy, the vacuum with the negative energy-spectrum (31) can be described as if a $\mathrm{N}$-floors building, two rooms each floor and all rooms occupied by guests; while the vacuum with the negative energy-spectrum (44) a $M$-floors $(M<N)$ building, $2 N / M$ rooms each floor and all rooms occupied by guests. The total numbers of rooms of two buildings are the same. Due to an external force, the $N$-floors building collapses to the $M$-floors building and the "potential energy" is reduced.

In principle, due to the vacuum state gains energy when the external magnetic field is applied upon it, the vacuum becomes unstable and must decay and release the energy $\Delta \mathcal{E}$ (12) by quantum-field fluctuations, analogously to the dynamics for the Casimir effect, discussed in the section of introduction. If the vacuum state decays and the vacuum energy 
(12) is released, the phenomena and effects that could occur are following. (i) The vacuum acts as a paramagnetic medium that effectively screens the strength of the external magnetic field $B$ to a smaller value $B^{\prime}<B$ for the total energy-density being,

$$
\frac{1}{2} B^{\prime 2}=\frac{1}{2} B^{2}-8 \frac{\alpha}{3 \pi} B^{2} ; \quad B^{\prime}=B \sqrt{1-\frac{16}{3 \pi} \alpha} .
$$

This phenomenon of paramagnetic screening could be possibly checked by appropriate experiments of precisely measuring the magnetic field strength. (ii) The vacuum-energy fluctuations could lead to the emission of neutrino and anti-neutrino pairs from the vacuum, since they are almost massless. This however is almost impossible for an experimental test. (iii) Photons are spontaneously emitted analogously to the spontaneous photon emissions for electrons at high-energy levels decaying to low-energy levels in the atomic physics. This phenomenon should be possibly detected if any photons are emitted when the magnetic field $B$ is turned on.

In practice, it must be very complicate to measure this vacuum energy $\Delta \mathcal{E}$ (12) releasing. In the following section, we discuss the rate and spectrum of spontaneous photon emissions.

\section{THE RATE AND SPECTRUM OF SPONTANEOUS PHOTON EMISSIONS}

Let us assume the magnetic field $B=B(t)$ is adiabatically turned on

$$
B(t)= \begin{cases}0, & t=t^{-} \rightarrow-\infty \\ B, & t=t^{+} \rightarrow+\infty\end{cases}
$$

in the time interval $\Delta \tau=t^{+}-t^{-}$. Based on this assumption, we compute rate and spectrum of spontaneous photon emissions, purely due to the variation of vacuum energy (12).

In order to obtain the rate and spectrum of spontaneous photon emissions, we need to compute a transition amplitude from the "initial" fermionic vacuum state $(B=0)$ to the "final" fermionic vacuum state $(B \neq 0)$, i.e., from initial negative energy-states $\psi_{i}^{(-)}$of fermions when $B=0$ to final negative energy-states $\psi_{f}^{(-)}$of fermions when $B \neq 0$. For simplifying computations of the transition amplitude at tree-level, we consider that the initial negative energy-states $\psi_{i}^{(-)}$of fermions are zero-momentum states $\left(\vec{p}_{i}=0\right)$, and the final negative energy-states $\psi_{f}^{(-)}$of fermions are all possible states. Beside, we chose the magnetic field $B$ is in $\hat{z}$-direction and the electric charge $e$ is renormalized. Thus, the initial 
negative energy-states $\psi_{i}^{(-)}$for $B=0$ and final negative energy-states $\psi_{f}^{(-)}$for $B \neq 0$ are given by

$$
\begin{aligned}
\psi_{i}^{(-)} & =\left(\frac{1}{V} \frac{m}{E_{i}}\right)^{\frac{1}{2}} e^{i E_{i} t}\left(\begin{array}{c}
0 \\
\chi^{\alpha}
\end{array}\right) ; \\
\psi_{f}^{(-)} & =\left(\frac{1}{V} \frac{m}{E_{f}}\right)^{\frac{1}{2}} c_{n} e^{-\frac{\xi^{2}}{2}} H_{n}(\xi) e^{i E_{f} t-p_{y}^{f} y-p_{z}^{f} z}\left(\begin{array}{c}
0 \\
\chi^{\alpha}
\end{array}\right) \\
E_{i} & =m, \\
E_{f} & =\sqrt{m^{2}+\left(p_{z}^{f}\right)^{2}+e B(2 n+1-\alpha)},
\end{aligned}
$$

where the spinor $\chi^{\alpha}$ : $\sigma_{z} \chi^{\alpha}=\alpha \chi^{\alpha}$ for the helicity $\alpha= \pm 1, \xi=\sqrt{e B}\left(x-\frac{p_{y}^{f}}{e B}\right), H_{n}(\xi)$ is the Hermite polynomial and $c_{n}=1 /\left(2^{\frac{n}{2}} \sqrt{n !} \pi^{\frac{1}{2}}\right)$. These negative energy solutions can be obtained by the charge conjugation of corresponding positive energy-solutions. The probability of spontaneous photon emissions is related to the amplitude $\left|\epsilon_{\mu}^{\beta} J^{\mu}(k)\right|^{2}[9]$, where $\epsilon_{\mu}^{\beta}$ is the transverse polarization vector of photons emitted and

$$
J^{\mu}(k)=e \int d^{4} x e^{-i k x} \bar{\psi}_{f}^{(-)} \gamma^{\mu} \psi_{i}^{(-)},
$$

where " $k$ " is photon's energy-momentum. Using $\psi_{i}^{(-)}$(15) and $\psi_{f}^{(-)}$(16), we integrate variables $t, y$ and $z$ in Eq.(19), which gives rise to $\delta$-functions for energy-momentum conservations. Armed with Eq.(7.376) in [7], we integrate variable $x$ in Eq.(19),

$$
\int d x e^{-i k_{x} x} e^{-\frac{\xi^{2}}{2}} H_{n}(\xi)=(-i)^{n}\left(\frac{2 \pi}{e B}\right)^{\frac{1}{2}} e^{-i k_{x} \frac{p_{y}^{f}}{e B}} H_{n}\left(\frac{k_{x}}{\sqrt{e B}}\right) e^{-\frac{k_{x}^{2}}{2 e B}} .
$$

The computation of the amplitude $\left|\epsilon_{\mu}^{\beta} J^{\mu}(k)\right|^{2}$ is straightforward in the spinor space. Taking average over helicities of initial states, summing over all final states $p_{z}^{f}, p_{y}^{f}$ and $n$ with degeneracy $|e| B S /(2 \pi)$, as well as the polarizations of photons emitted, we obtain,

$$
\begin{aligned}
\frac{1}{2} \sum_{\beta}\left|\epsilon_{\mu}^{\beta} J^{\mu}(k)\right|^{2} & =e^{2} e^{-\frac{k_{x}^{2}}{e B}} \sum_{n=1}^{\infty} \frac{1}{2^{n} n ! \pi} H_{n}^{2}\left(\frac{k_{x}}{\sqrt{e B}}\right)(2 \pi) \delta\left[\omega_{k}-E_{f}^{n}+m\right]\left(\frac{m}{E_{f}^{n}}\right) \Delta \tau \\
E_{f}^{n} & =\sqrt{m^{2}+\left(k_{z}\right)^{2}+2 e B n}
\end{aligned}
$$

where $\omega_{k}=|k|$ is the photon energy and $\delta$-function for the energy-conservation. The term corresponding to $n=0$ has been dropped for energy-momentum conservations, since the $\delta$-function $\delta\left[\omega_{k}-E_{f}^{n=0}+m\right]$ only gives solution $|k| \equiv 0$. Because the problem is axial symmetric w.r.t. $\hat{z}$-direction, we can make substitutions $k_{x}^{2} \rightarrow k_{\perp}^{2}=k_{x}^{2}+k_{y}^{2}, k_{x} \rightarrow\left|k_{\perp}\right|$ and 
define $k_{z}=k_{\|}$in Eq.(21). The $\delta$-function in Eq.(21) can be given as

$$
\delta\left[\omega_{k}-E_{f}^{n}+m\right]=\left(\frac{m+|k|}{e B}\right) \delta_{n, n_{\circ}}, n_{\circ}=\frac{k_{\perp}^{2}+2|k| m}{2 e B}, \quad E_{f}^{n_{\circ}}=m+|k|,
$$

where $n_{\circ}=1,2,3, \cdots$, indicating the energy-momentum of emitted photons is quantized. As a result, Eq.(21) is,

$$
\frac{1}{2 \Delta \tau} \sum_{\beta}\left|\epsilon_{\mu}^{\beta} J^{\mu}(k)\right|^{2}=\frac{e^{2}}{2^{n_{\circ}} n_{\circ} ! \pi} e^{-\frac{k_{\perp}^{2}}{e B}} H_{n_{\circ}}^{2}\left(\frac{\left|k_{\perp}\right|}{\sqrt{e B}}\right)(2 \pi) \sum_{f}\left(Q_{f}^{2}\right)\left(\frac{m_{f}}{e B}\right),
$$

where $\sum_{f}$ is over all flavors of charged fermions. We find that Eq.(23) does not explicitly depend on $k_{\|}$. For given $n_{\circ}=n_{\circ}\left(k_{\perp}, k_{\|}\right)$in (22), Eq.(23) (the probability of photon emissions) is very small for $\left|k_{\|}\right| \gg\left|k_{\perp}\right|$, because the polynomial $H_{n_{\circ}}^{2}\left(\frac{\left|k_{\perp}\right|}{\sqrt{e B}}\right)$ in Eq.(23) is very small for small values of $\left|k_{\perp}\right|$. Thus, the most probability of spontaneous photon emissions occurs for $\left|k_{\perp}\right| \gg\left|k_{\|}\right|$, indicating most emitted photons are near in the plane perpendicular to the magnetic field $B$. This is analogous to the phenomenon of synchrotron radiation.

The probability $p_{n_{\gamma}}$ corresponding to the emission of $n_{\gamma}$ photons, when neither the momentum nor the polarizations are observed, is given by the Poisson distribution[9],

$$
p_{n_{\gamma}}=\frac{\bar{n}_{\gamma}}{n_{\gamma} !} e^{-\bar{n}_{\gamma}}
$$

where $\bar{n}_{\gamma}$ is defined by

$$
\bar{n}_{\gamma}=\int d \tilde{k} \frac{1}{2} \sum_{\beta}\left|\epsilon_{\mu}^{\beta} J^{\mu}(k)\right|^{2}, \quad \int d \tilde{k} \equiv \int \frac{d^{3} k}{(2 \pi)^{3} 2 \omega_{k}} \simeq \int\left(\frac{d k_{\|} d k_{\perp}}{(2 \pi)^{2} 2}\right)_{\omega_{k} \simeq\left|k_{\perp}\right|},
$$

which is actually the average number of emitted photons, $\bar{n}_{\gamma}=\sum_{\circ}^{\infty} n_{\gamma} p_{n_{\gamma}}$. The numberand energy-spectrum of spontaneous photon emissions in a phase space element $d \tilde{k}$ and a unit of time are given by

$$
\frac{d \bar{n}_{\gamma}}{d \tilde{k}}=\frac{1}{2 \Delta \tau} \sum_{\beta}\left|\epsilon_{\mu}^{\beta} J^{\mu}(k)\right|^{2}, \quad \frac{d \bar{\epsilon}_{\gamma}}{d \tilde{k}}=\frac{1}{2 \Delta \tau} \omega_{k} \sum_{\beta}\left|\epsilon_{\mu}^{\beta} J^{\mu}(k)\right|^{2},
$$

which are determined by Eq.(23).

We estimate that $\sqrt{e B} \simeq 0.244 \mathrm{eV}$ for $B=10^{5} \mathrm{G}$ achieved in the laboratory today 10$]$ and $\sqrt{e B} \simeq 24 \mathrm{KeV}$ for $B \simeq 10^{15} G$ around neutron stars, i.e., $\sqrt{e B} \ll m_{f}$. We consider the limit for emitted photons whose energy-momentum $|k| \ll m_{f}$. From Eq.(22), we have $n_{\circ}=$ $|k| m_{f} /(e B)$, i.e., $|k|=\omega_{k}=n_{\circ} e B / m_{f}$, showing the energy of emitted photons is quantized in the unit $e B / m_{f}$. As seen from Eq.(23), the probability is exponentially suppressed for large 
values of $k_{\perp}^{2} / e B$ and also suppressed by $1 /\left(2^{n_{\circ}} n_{\circ} !\right)$ for large values of $n_{\circ}$. As a consequence, most photons emitted should have the momentum $\left|k_{\perp}\right| \sim e B$ in the infrared region and quantized $\left|k_{\perp}\right| \simeq \omega_{k}=n_{\circ} e B / m_{f}$ for the small values of $n_{\circ}$. For $n_{\circ}=1,\left|k_{\perp}\right| \simeq|k|=\omega_{k}=$ $e B / m_{f}$ and $H_{1}(x)=2 x$, we have the rate,

$$
\frac{1}{2 \Delta \tau} \sum_{\beta}\left|\epsilon_{\mu}^{\beta} J^{\mu}(k)\right|^{2}=4 e^{2} e^{-\frac{k_{\perp}^{2}}{e B}} k_{\perp}^{2} \sum_{f}\left(Q_{f}^{2}\right) \frac{m_{f}}{(e B)^{2}} .
$$

for the number- and energy- spectrum (26) of spontaneous photon emissions. This spectrum shows $k_{\perp}^{2}$-dependence in the low-energy region and $\exp \left(-\frac{k_{\perp}^{2}}{e B}\right)$-dependence in the high energy region, respectively similar to the Rayleigh-Jeans part and the Wien part of the spectrum of the black-body radiation. However, the energy-momentum $\left|k_{\perp}\right| \simeq \omega_{k}$ is quantized, in this sense, it is more analogous to the Wigner spectrum of the distribution of discrete energylevels of atoms and nuclei.

This is a preliminary study of the possibilities of releasing vacuum energy $\Delta E$ (12). In reality, the way of releasing the vacuum energy $\Delta E$ must be complicated and the effect of the back-reaction to the vacuum should be considered. These are subjects for future work.

\section{POSSIBLY EXPERIMENTAL TEST}

Strictly speaking, the rate and spectrum of spontaneous photon emissions should depend on the way of turning on the magnetic field $B(t)$. The reason for us to make the assumption of adiabatical turning on magnetic field bases on the time-scale of quantum-field fluctuations of the vacuum is much smaller than $\Delta \tau$ (14). At this preliminary step, we regard the rate and spectrum of spontaneous photon emissions computed in the previous section as a theoretical analysis in the adiabatical assumption.

In practice, however, it must be difficult to measure the rate and spectrum of spontaneous photon emissions, purely attributed to the vacuum effect for the following reasons. (i) The variation of magnetic field with time must induce a variation of electric field with time, as a result, soft photons are emitted. These photons are hardly distinguished from photons spontaneously emitted due to the vacuum effect discussed in this article. (ii) The volume $(\Delta \tau \cdot c)^{3}$, in which vacuum states at different points of the space-time are causally-correlated, can be much larger than the volume $V$ in which the constant magnetic field is created. In addition, we know that the relaxation time of quantum-field fluctuations of vacuum states 
is of the order of $\hbar / m_{e} c^{2} \sim 10^{-20}$ sec. With this very short relaxation time, all causallycorrelated vacuum states at different points of the space-time can rapidly decay to the lower energy states before the maximum value $B$ of the external magnetic field is reached. In this case, we should not expect to detect spontaneous photon emissions only from the volume $V$, corresponding to the total vacuum-energy releasing given in Eq.(12), since photons can be spontaneously emitted from the whole volume $\sim(\Delta \tau \cdot c)^{3}$ where vacuum states are causally-correlated.

In the experiment of detecting the Casimir effect, two large parallel perfectly conducting plates of sizes $L^{2}$ at a distance $a(L \gg a)$ separate causal-correlation of the quantumfield fluctuations of virtual photons inside the volume $a L^{2}$ between two plates from the quantum-field fluctuations of virtual photons outside of two plates. Since the creations and annihilations of virtual fermions and anti-fermions are related to the annihilation and creation of virtual photons, we assume the causal-correlation between vacuum states at two different points of the space-time is mediated by virtual photons. Inspired by the experiment for detecting the Casimir effect, we should use perfectly conducting box to isolate the volume $V$, where the external magnetic field is adiabatically turned on, from the space-time outside the box. The photon monitor and instrument of measuring magnetic field strength should be properly installed inside the box to detect the possible effect of spontaneous photon emissions. It is obvious that much sophisticate experiments must be proposed and/or another brilliant ideas for experiments have to be created, in order to detect such effects.

Though such vacuum effects are difficult to be measured in a ground Laboratory, they could be probably observed in astrophysics events of photon and neutrino emissions, due to a very large variation of strength of magnetic fields in astrophysics processes (e.g., supernova explosions and neutron stars). Given the size of a neutron star of the order $10^{6} \mathrm{~cm}$ and the strength of magnetic fields of the order of $10^{13}-10^{15} \mathrm{G}$, we can estimate the maximum total vacuum-energy releasing is of the order of $10^{42}-10^{46} \mathrm{erg}$ from Eq.(12). It is worthwhile to mention that such vacuum effects could account for the anomalous X-ray pulsar [1]].

Rotating Kerr black holes also can possess a large variation of the strength of magnetic fields, the vacuum effects discussed in this article can take place around the black hole. We do not know that any phenomenon relating to these vacuum effects can be observable. Regarding the applications of these vacuum effects to astrophysical processes of neutron stars 
and black holes, we have to consider whether or not the effect of gravitational field should be taken into account. If the gravitational field is significantly strong enough, the vacuum

effects should be modified, see for example [13]. In the neutron star case of size $10^{6} \mathrm{~cm}$, mass one solar mass and magnetic field $10^{13} \mathrm{G}$, we can estimate that the force of gravitational field acting on an electron is about $2 \cdot 10^{-12}$ Newton, while the force of magnetic field acting on an electron is about 5 Newton. This implies that the gravitational field effects on the vacuum effects can be negligible in the neutron star cases. However, in the Kerr black hole case, the effects of gravirational field on the black hole's horizon must not be negligible. It is worthwhile to study such gravitational effects.

\section{CONCLUSION}

Before ending this article, we wish to clarify that the effect presented here is essentially different from the well-known Heisenberg-Euler-Schwinger process 12] on the view point of dynamics, though both of them are related to an external electromagnetic field. Let us first recall the main points of the Heisenberg-Euler-Schwinger process.

The quantum-field fluctuations of virtual particles and antiparticles are rather perturbative, for their space and time variations being much smaller than $\hbar / m c$ and $\hbar / m c^{2}$. The vacuum is vary stable against such small quantum-field fluctuations of virtual electrons and positrons (and other fermions), for the reasons: (i) all negative energy-states are fully filled; (ii) the energy-gap $2 m$ is the potential barrier to block small quantum-field fluctuations leading to a vacuum instability. The probability of the vacuum breakdown and decaying by the pair-creation of real electrons and positrons (the probability of tunneling the barrier) is exponentially suppressed, practically is zero. The external electric field applied upon the vacuum effectively reduces the energy-gap of barrier and the vacuum is polarized along the direction the electric field. When the electric field is increased to the critical field $E_{c} \simeq m^{2} c^{3} / e \hbar$, the probability of virtual electrons and positron tunneling the barrier significantly increases, leading to the phenomenon of the vacuum breakdown by pair-creation of electrons and positrons in the Heisenberg-Euler-Schwinger process.

It is important to notice that the Heisenberg-Euler-Schwinger process is a purely electric effect from the dynamics point of view: the electric field instead of magnetic field makes the vacuum decaying and pair-production be feasible. On the contrary, the effect presented 
here is purely magnetic. As discussed in sections (IIIIII), the dynamics of inducing vacuum decaying is completely due to the fact that the external magnetic field modifies the negative energy-spectrum from non-degenerate one to degenerate one, as a result shifts the negative energy of the vacuum downwards, rather than reduces the energy-gap $2 m$ separating the negative energy-spectrum from the positive energy-spectrum. In contrast, the electric field in the Heisenberg-Euler-Schwinger process does not change negative energy-spectrum of the vacuum. The possible phenomena of the presented effect are screening magnetic field and spontaneous photon emissions, instead of pair-production of electrons and positions. In principle, the presented effect can occur for any value of magnetic field and non critical value of field is required. This is very different from the critical electric field in the HeisenbergEuler-Schwinger process. In fact, the dynamics of this effect presented here is analogous to the dynamics of the Casimir effect, rather than the dynamics of the Heisenberg-EulerSchwinger one. The situation somehow resembles the phenomenon of chiral gauge anomalies: chiral fermions are derived out of the Dirac sea (vacuum) by a chiral gauge field, leading to anomalous particles production.

In summary, we propose an idea of releasing vacuum-energy from the vacuum by introducing an external magnetic field. This idea is originated from (i) the fermionic structure of the vacuum owing to quantum field theories and the Pauli principle; (ii) the external magnetic field modifying the fermion spectrum of the vacuum from non-degenerate one to degenerate one; (iii) the quantum-field fluctuations of virtual particles in the vacuum must lead the vacuum state to the lowest energy-state. We illustrate this idea by giving an explicit computation of vacuum energy and showing the vacuum-energy releasing is about one percent of the total energy stored in the external magnetic field. We point out that such a vacuum-energy releasing could be realized by paramagnetic screening and/or spontaneous photon emissions. We compute the possible rate and spectrum of spontaneous photon emissions. In addition, we discuss the difficulties to observe such vacuum effects and propose a possible experiment to detect these effects. In today's laboratory, the magnetic field strength has been reached up to (greater than) $10^{5} \mathrm{G}$ and large stored energy up to (larger than) tens or hundreds of MJ [10]. We expect a sophisticate experiment in near future to verify the phenomena and effects of the vacuum-energy releasing via paramagnetic screening and spontaneous photon emissions induced by external magnetic fields. This is important for the understanding of the fermion structure of the vacuum of quantum field theories and 
any possibly prospective applications.

[1] W. E. Lamb and R. C. Retherford, Phys. Rev. vol. 72 (1947) 241.

[2] H.B.G. Casimir, Proc. Kon. Ned. Akad. Wetenschap., ser. B, vol. 51, p. 793, 1948. see also M. Fierz, Helv. Phys. Acta, vol. 33, p. 855, 1960.

[3] M.J. Sparnnaay, Physica, vol. 24, p. 751, 1958;

S.K. Lamoreaux, Phys. Rev. Lett. 78 (1957) 5; U. Mohideen and A. Roy, ibid 81 (1998) 4549.

[4] D. Landau and M. Lifsits, "Theory of relativistic quantum mechanics" (1975) p.357.

[5] G. 't Hooft and M. Veltman, Nucl. Phys. B44 (1972) 189.

[6] J.S. Dowker and R. Banach (1978) J. Phys. A: Gen. Phys., 11, 2255.

[7] I.S. Gradshteyn and I.M. Ryzhik, "Table of integrals, series, and products" (1965) New York Academic Press.

[8] S. K. Blau, M. Visser and A. Wipf, Int. J. Mod. Phys. A6 (1991) 5409-5433. Thanks to the referee for bring this reference into author's attention.

[9] C. Itzykson and J.-B. Zuber, "Quantum Field Theory" 1980 McGraw-Hill Inc.

[10] see 'http://www.nhmfl.gov/magtech/lhfs/index.html'.

[11] G.L. Israel, T. Oosterbroek, L. Stella, S. Campana, S. Mereghetti and A.N. Parmar astro-ph/0108506, ApJ Letters (in press).

[12] W. Heisenberg and H. Euler, Z. Physik vol. 98 (1936) 714;

J. Schwinger, Phys. Rev., vol 82, (1951) 664.

[13] B. Geyer and S. D. Odintsov, Phys. Rev D53, 1996, 7321 and Int. J. Mod. Phys. A11,1996,2053. 